Journal of Animal and Veterinary Advances $10(7): 841-846,2011$

ISSN: $1680-5593$

(C) Medwell Journals, 2011

\title{
Quantitative Trait Gene Responsible for Intramuscular Fat Content in the Rat
}

\author{
${ }^{1}$ Hideki Tanomura, ${ }^{1}$ Takuji Yamamoto, ${ }^{2}$ Youji Muramatsu, ${ }^{3}$ Takeshi Ohta and ${ }^{1}$ Takahisa Yamada \\ ${ }^{1}$ Laboratory of Animal Genetics, Graduate School of Science and Technology, \\ Niigata University, Nishi-Ku, 950-2181 Niigata, Japan \\ ${ }^{2}$ Department of Nutritional Sciences for Well-being, Faculty of Health Sciences for Welfare, \\ Kansai University of Welfare Sciences, Kashiwara, 582-0026 Osaka, Japan \\ ${ }^{3}$ Central Pharmaceutical Research Institute, Japan Tobacco, Inc., \\ Takatsuki, 569-1125 Osaka, Japan
}

\begin{abstract}
Marbling defined by the amount and distribution of intramuscular fat, socalled Shimofuri is an economically important trait of beef cattle in Japan. A rat Quantitative Trait Locus (QTL) responsible for intramuscular fat content has been mapped to the $\sim 10$-cM genomic region on chromosome 1 by utilizing the Otsuka Long-Evans Tokushima Fatty (OLETF) rat and OLETF-derived congenic strain. In this study, we have attempted to identify the Quantitative Trait Gene (QTG) for the intramuscular fat content QTL by using the OLETF rat. For this purpose, we performed refinement of the intramuscular fat content QTL genomic region to a $\sim 2.3$-cM region by use of informative recombinants selected from the OLETF-derived congenic strain, functional retrieval of positional candidate gene, mRNA level analysis and polymorphism search of physiologically relevant positional candidate gene, Pnlip and analysis of cosegregation of the intramuscular fat content QTL with Variable Number of Tandem Repeat (VNTR) in Pnlip by use of informative recombinants. This study suggests that Pnlip encoding pancreatic lipase is the QTG for the intramuscular fat content QTL.
\end{abstract}

Key words: Candidate gene, intramuscular fat, marbling, QTG, QTL, rat

\section{INTRODUCTION}

Generally, marbling means the amount of intramuscular fat (Cameron et al., 1994). In Japan, marbling is characterized as the amount and distribution of intramuscular fat in a cross section of Musculus longissimus muscle and is called Shimofuri. High levels of such marbling improve the palatability and acceptability of beef by affecting the taste and tenderness of the meat (Busboom et al., 1993; Boylston et al., 1995). Because of the importance of marbling on the economics of beef production, there is great interest in gaining a better understanding of the molecular architecture of marbling that is intramuscular fat content.

The Otsuka Long-Evans Tokushima Fatty (OLETF) rat, an animal model for obese type 2 diabetes, exhibits higher levels of intramuscular fat content in Musculus longissimus than the F344 rat (Tanomura et al., 2002). We previously reported that a genomic region between D1Wox8 and D1Rat90 on chromosome 1 influences intramuscular fat content with the OLETF allele acting on an increase in fat content by Quantitative Trait Locus
(QTL) analysis using $\mathrm{F}_{2}$ progenies derived from the OLETF and F344 rats (Tanomura et al., 2002). Further, we demonstrated that the QTL on chromosome 1 responsible for intramuscular fat content is located in the $\sim 10-\mathrm{cM}$ genomic region between D1Rat166 and D1Rat90 using a congenic strain trapping the OLETF allele of the $\sim 10-\mathrm{cM}$ region on the $\mathrm{F} 344$ genetic background in a monogenic context (Tanomura et al., 2003).

The present study has attempted to identify the Quantitative Trait Gene (QTG) for the intramuscular fat content QTL by utilizing the OLETF rat. For this purpose, we performed refinement of the intramuscular fat content QTL genomic region to a $\sim 2.3-\mathrm{cM}$ region by use of informative recombinants selected from the congenic strain, functional retrieval of positional candidate gene, mRNA level analysis and polymorphism search of physiologically relevant positional candidate gene, Pnlip and analysis of cosegregation of the intramuscular fat content QTL with Variable Number of Tandem Repeat (VNTR) in Pnlip by use of informative recombinants. This study suggests that Pnlip encoding pancreatic lipase is the QTG for the intramuscular fat content QTL.

Corresponding Author: Takahisa Yamada, Laboratory of Animal Genetics, Graduate School of Science and Technology, Niigata University, Nishi-ku, 950-2181 Niigata, Japan 


\section{MATERIALS AND METHODS}

Animals: The researchers used the inbred strains of OLETF (OLETF/Otk) (Kawano et al., 1992), normal F344 (F344/Crj) and normal BN rats (BN/Tj). We also used the congenic strain constructed by introgressing the OLETF allele on the $\sim 10-\mathrm{cM}$ intramuscular fat content QTL region between D1Rat166 and D1Rat90 into the normal F344 rat strain (Tanomura et al., 2003). Furthermore, researchers constructed informative recombinants in which a part of the intramuscular fat content QTL region between D1Rat166 and D1Rat90 in the congenic strain was replaced by the F344 background genome, to refine the intramuscular fat content QTL region. For breeding of informative recombinants, the $F_{1}$ rats were generated by female congenic rats bred with male F344 rats and male backcross progenies were obtained by mating the female $\mathrm{F}_{1}$ with male congenic rats.

While the male OLETF rat possesses $~ 2.4$-fold higher levels of intramuscular fat content in Musculus longissimus than male F344 rat, no difference in the levels is detected between OLETF and F344 females (Tanomura et al., 2002). Thus, using males was assumed to facilitate exclusively the detection of the quantitative difference of intramuscular fat content. All rats were kept under specific pathogen-free conditions. The temperature $\left(21 \pm 2^{\circ} \mathrm{C}\right)$, humidity $(55 \pm 5 \%)$ and air conditioning were all controlled.

Rats had free access to tap water and standard laboratory chow (CRF-1; Oriental Yeast, Tokyo, Japan) and were maintained on a $12 \mathrm{~h}$ light and dark cycle (6 a.m./6 p.m.). This study conformed to the guidelines for animal experimentation of the Graduate School of Science and Technology, Niigata University (Niigata, Japan).

Genotyping: Male rat tails were cut and frozen at $-80^{\circ} \mathrm{C}$ for subsequent isolation of high-molecular-weight DNA. Genotyping of D1Rat166 and D1Rat90 with tail biopsy DNA of male backcross progenies was performed as described previously (Tanomura et al., 2003), to select male backcross progenies which harbored heterozygote at either one of D1Rat166 or D1Rat90 and homozygote at the other as informative recombinants (Table 1). Subsequently, genotyping of D1Rat225 located on a genomic interval between D1Rat166 and D1Rat90 (Wei et al., 1998) was performed for the informative recombinants by PCR with primers purchased from Research Genetics (Huntsville, AL) according to a previous report (Wei et al., 1998) to determine a refined region of the intramuscular fat content QTL (Table 1).

Phenotype evaluation: Male rats were sacrificed under deep anesthesia at 35 weeks of age. The body weight was
Table 1: Genotyping result and phenotype evaluation in 12 informative recombinants selected from (congenic $\times$ F344) $F_{1} \times$ congenic backcross progenies

\begin{tabular}{lcccc}
\hline \multicolumn{5}{c}{ Genotype } \\
Phenotypes & No. of animals & $\begin{array}{c}\text { D1Rat166 } \\
(0-\mathrm{cM})\end{array}$ & $\begin{array}{c}\text { D1Rat225 } \\
(4.5-\mathrm{cM})\end{array}$ & $\begin{array}{c}\text { D1Rat90 } \\
(6.8-\mathrm{cM})\end{array}$ \\
\hline Low level & 4 & $\mathrm{O} / \mathrm{O}$ & $\mathrm{O} / \mathrm{F}$ & $\mathrm{O} / \mathrm{F}$ \\
& 1 & $\mathrm{O} / \mathrm{O}$ & $\mathrm{O} / \mathrm{O}$ & $\mathrm{O} / \mathrm{F}$ \\
High level & 4 & $\mathrm{O} / \mathrm{F}$ & $\mathrm{O} / \mathrm{O}$ & $\mathrm{O} / \mathrm{O}$ \\
& 2 & $\mathrm{O} / \mathrm{F}$ & $\mathrm{O} / \mathrm{F}$ & $\mathrm{O} / \mathrm{O}$ \\
& 1 & $\mathrm{O} / \mathrm{O}$ & $\mathrm{O} / \mathrm{O}$ & $\mathrm{O} / \mathrm{F}$ \\
\hline
\end{tabular}

$\mathrm{O} / \mathrm{O}$ and $\mathrm{O} / \mathrm{F}$ represent homozygosity for the OLETF allele and heterozygosity, respectively. Relative genetic map positions of D1Rat166, D1Rat225 and D1Rat90 on rat chromosome 1 are shown in parentheses

measured before the sacrifice of rats. Immediately after exsanguination, the abdominal fat pads consisting of mesenteric, retroperitoneal and epididymal fat pads were removed and weighed. Furthermore, the first to third lumbar region of Musculus longissimus was removed from the right side of the carcass and rapidly frozen in dry ice-cooled isopentane. Five serial coronal sections ( $6 \mu \mathrm{m}$ thick) were obtained at intervals of $180 \mu \mathrm{m}$ from frozen Musculus longissimus and the sections were stained with Oil Red O to detect the presence of lipid droplets in adipocyte cytoplasm. The section area, occupied by cells positive for Oil Red O staining were measured in a given area $\left(1 \mathrm{~cm}^{-2}\right)$ using the MacSCOPE computer program. The fat staining-positive area expressed as an absolute value was used as the phenotypic value of the level of intramuscular fat content (Tanomura et al., 2003).

Pnlip mRNA level analysis: The pancreases were removed after overnight starvation from male rats at 15 and 35 weeks of age and frozen at $-80^{\circ} \mathrm{C}$. Total RNAs were prepared from the pancreases using ISOGEN (WAKO Pure Chemical Industries, Osaka, Japan). Firststrand cDNA was reverse-transcribed from total RNA sample $(1 \mu \mathrm{g})$ using RNA PCR Kit ver. 2.1 (Takara Bio, Shiga, Japan). Competitive PCR $\mathrm{w}$ as performed for Pnlip mRNA level analysis using reverse-transcribed reaction mixture as described previously (Shikamoto and Morita, 1999). PCR primers used in competitive PCR were designed from the rat cDNA sequences of Pnlip (GenBank accession number NM_013161) and glyceraldehyde-3-phosphate dehydrogenase gene (Gapdh) (GenBank accession number NM_017008). Primer sequences for Pnlip were 5'-ATACACCCAGGCTAC CCA GAAC-3' (nucleotide positions 419-440 of NM_013161) and 5'-CCAATAGCTCCGAATGTCCTCT-3' (nucleotide positions 589-568 of NM_013161). Primer sequences for Gapdh were 5'-TCCTGCACCACCAAC TGCTTA-3' (nucleotide positions 1294-1314 of NM_017008) and 5'-ACCACCCTGTTGCTGTAGCCA-3' (nucleotide 
positions 1817-1797 of NM_017008). DNA competitors used in competitive PCR were generated by PCR with PCR composite primers which were engineered to contain the competitor template-specific sequences with the target gene-specific primer sequences, using Competitive DNA Construction Kit (Takara Bio). Sequences of PCR composite primers for Pnlip competitor were 5'ATACACCCAGGCTACCCAGAACGTAC GGTC ATCATCTGACAC-3' (nucleotide positions 419-440 of NM_013161 plus 1-20 of competitor template) and 5'CCAATAGCTCCGAATGTCCTCTTCATTACGCATCG CTATTAC-3' (nucleotide positions 589-568 of NM_013161 plus 200-181 of competitor template). Sequences of PCR composite primers for Gapdh competitor were 5'-TCCT GCAC CACCAA CTG CTT A GTACGGTCATCATCTGACAC-3' (nucleotide positions 1294-1314 of NM_017008 plus 1-20 of competitor template) and 5'-ACCACCCTGTTGCTGTAGCCAGCGTGAGT ATTACGAAGGTG-3' (nucleotide positions 1817-1797 of NM_017008 plus 400-381 of competitor template). Concentration of DNA competitors was measured by spectrophotometry $\left(\mathrm{A}_{260}\right)$ and subsequently adjusted to $1 \times 10^{11}$ copies $\mu \mathrm{L}$. A series of competitive PCR was performed with $0.5 \mu \mathrm{M}$ of each PCR primer, DNA competitor ( 10 fold serial dilution from $10^{1}-10^{10}$ copies/reaction) and $0.1 \mu \mathrm{L}$ reverse-transcribed reaction mixture, using RNA PCR Kit ver. 2.1 (Takara Bio).

A thermocycling protocol was 30 cycles of denaturation for $45 \mathrm{sec}$ at $94^{\circ} \mathrm{C}$, annealing for $45 \mathrm{sec}$ at $60^{\circ} \mathrm{C}$ and elongation for $45 \mathrm{sec}$ at $72^{\circ} \mathrm{C}$. PCR products were separated by electrophoresis on a 3.0\% Metaphor agarose gel (Takara Bio). Agarose gels were stained with ethidium bromide and photographed under an ultraviolet light. Digital images of photographs were imported into the Scion Image computerized densitometry program (Scion, Frederick, MD).

cDNA copy numbers of Pnlip and Gapdh were calculated according to the method previously reported (Shikamoto and Morita, 1999). Briefly, intensity ratio of target DNA bands to corresponding competitor DNA bands was plotted on a logarithmic scale against copy number of competitors and then an equivalence point $(\log$ ratio $=0)$ was calculated from a linear regression standard curve. Results of competitive PCR for Gapdh were used for normalization of cDNA amounts subjected to competitive PCR for Pnlip.

Pnlip polymorphism search: Pnlip polymorphisms between the F344 and OLETF rats were searched by the direct sequencing. About 95 pairs of PCR primers were designed to cover $\sim 26.6-\mathrm{kb}$ Pnlip genomic sequences (nucleotide positions 1788220-1814849 of GenBank accession number NW_047570) which included the
5 'flanking region $(\sim 9.8-\mathrm{kb})$, the exon/intron region $(\sim 13.1-\mathrm{kb})$ and the 3 '-flanking region $(\sim 3.6-\mathrm{kb})$. PCR was carried out with $0.5 \mu \mathrm{M}$ of each PCR primer and genomic DNA (100 ng) isolated from tails of the F344 and OLETF rats, using TaKaRa Ex Taq (Takara Bio). A thermocycling protocol was the same as described in the mRNA level analysis section. DNA sequencing of PCR products was performed by the direct sequencing with capillary DNA sequencer RISA-384 system in Shimadzu Corporation (Kyoto, Japan). GENETYX-WIN3.1 program (Software Development, Tokyo, Japan) was used for analysis of DNA sequence.

Genotyping of Pnlip VNTR: Pnlip VNTR was genotyped with tail biopsy DNA by PCR amplification of $\mathrm{a} \sim 2.8-\mathrm{kb}$ genomic segment which contains tandem repeats of 38-bp core sequence between the bases 5517 and 2698 upstream from the transcription start site of Pnlip (nucleotide positions 1792587-1795406 of NW_047570). Primer sequences were 5'-TGTGCAGAGCACTGCGTCAC3 ' (nucleotide positions 1792587-1792606 of NW_047570) and 5'-CACAGTGCTCTGCTTGTGGA-3' (nucleotide positions 1795406-1795387 of NW_047570). PCR was carried out as described in the polymorphism search section.

A thermocycling protocol was 30 cycles of denaturation for $60 \mathrm{sec}$ at $94^{\circ} \mathrm{C}$, annealing for $60 \mathrm{sec}$ at $60^{\circ} \mathrm{C}$ and elongation for $180 \mathrm{sec}$ at $72^{\circ} \mathrm{C}$. PCR products were electrophoresed on a $1.0 \%$ agarose gel (Takara Bio).

Statistical analysis: Comparisons of phenotypes and Pnlip mRNA levels among different genotypic groups were performed by an Analysis of Variance (ANOVA) with a post hoc test using Scheffe's F test (StatView; SAS Institute, Gary, NC).

\section{RESULTS AND DISCUSSION}

Phenotype evaluation in congenic and (congenic $x$ F344) $\mathbf{F}_{1}$ rats: The level of intramuscular fat content was increased and decreased in the congenic rat as compared with the F344 and OLETF rats, respectively and both the differences in the changes were statistically significant $(\mathrm{p}<0.05)$ (Fig. 1). This result of an increase in the level of intramuscular fat content of the congenic strain over the F344 rat was consistent with the previous finding (Tanomura et al., 2003). Additionally, the level in (congenic female x F344 male) $F_{1}$ rat was intermediate between those of the F344 and the congenic rats and all the differences between the $F_{1}$ and the F344 or congenic were statistically significant $(\mathrm{p}<0.05)$ (Fig. 1). This result indicates that the OLETF allele at the intramuscular fat 


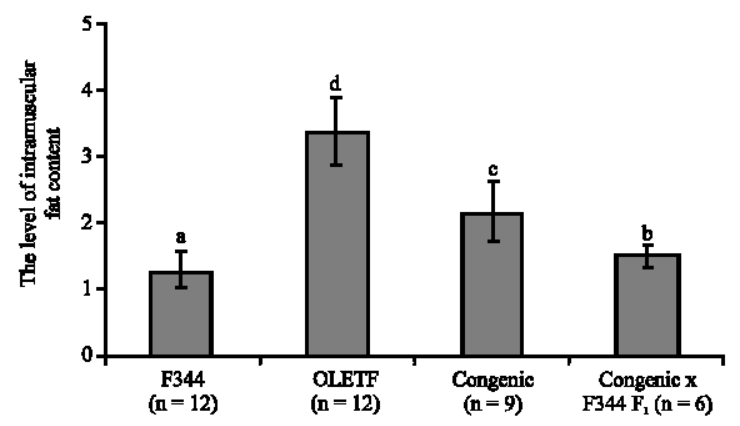

Fig. 1: The level of intramuscular fat content in F344 $(\mathrm{n}=12), \operatorname{OLETF}(\mathrm{n}=12)$, congenic $(\mathrm{n}=9)$ and (congenic $x$ F344) $F_{1}$ rats $(n=6)$. Only male rats were phenotyped at 35 weeks of age. Columns and bars represent means and $\mathrm{SDs}$, respectively. Comparisons of the level among the four genotypic groups were performed by one-way analysis of variance with Scheffe's F analysis as a post hoc test. ${ }^{\text {add }}$ Means without a common letter in their superscripts differ $(\mathrm{p}<0.05)$

content QTL acts on an incrase of intramuscular fat content via an additive mode of inheritance, inconsistent with the result (via a recessive mode of inheritance) of QTL analysis in (OLETF $x$ normal) $F_{2}$ intercross (Tanomura et al., 2002). The reason for this inconsistency is unclear.

Refinement of the genomic region of the intramuscular fat content QTL: We have constructed 176 male (congenic $\mathrm{x}$ F344) $\mathrm{F}_{1} \mathrm{x}$ congenic backcross progenies. Among them, 12 backcross progenies harbored heterozygote at either one of D1Rat166 or D1Rat90 and homozygote at the other and thus were selected as informative recombinants in which a part of the intramuscular fat content QTL region between D1Rat166 and D1Rat90 in the congenic strain was replaced by the F344 background genome (Table 1).

The distribution of the level of intramuscular fat content among the 12 progenies allowed the informative recombinants to divide clearly into 2 groups, high level and low level groups, phenotypic values of which were similar to those of the congenic and (congenic $x$ F344) $F_{1}$ rats, respectively.

Thus, the high level and low level groups were assigned as OLETF allele homozygote at the intramuscular fat content QTL $(\mathrm{O} / \mathrm{O}, \mathrm{n}=7)$ and heterozygote $(\mathrm{O} / \mathrm{F}, \mathrm{n}=5)$, respectively (Table 1$)$. Subsequent genotyping of D1Rat225 for the informative recombinants refined the intramuscular fat content QTL genomic region to a $\sim 2.3-\mathrm{cM}$ region between D1Rat225 and D1Rat90 (Table 1).
Functional retrieval of positional candidate gene: About 46 genes were detected within the $\sim 2.3-\mathrm{cM}$ intramuscular fat content QTL region on the rat genome (rat genome resources). Functional retrieval of the 46 genes revealed that Pnlip which encodes pancreatic lipase involved in energy income and fat regulation was regarded as the most prominent and physiologically relevant positional candidate gene for the intramuscular fat content QTL. Pnlip is composed of 13 exons spanning $>13-\mathrm{kb}$ and encodes a $56-\mathrm{kD}$ protein of 465 amino acid residues (Sims et al., 1993).

The hydrolysis of dietary triglycerides to fatty acids by pancreatic lipase encoded by Pnlip is essential for the intestinal absorption of long-chain triglyceride fatty acids (Lowe, 2002), indicating an involvement of pancreatic lipase in energy income. Further, the treatment with Orlistat that blocks predominantly pancreatic lipase activity in the small intestine promotes significant fat loss and prevents fat regain in obese patients (Sjostrom et al., 1998; Hill et al., 1999) indicating an involvement of pancreatic lipase in fat regulation. Thus, an increase in pancreatic lipase amounts is likely to result in excess energy income and then increase of intramuscular fat content.

Pnlip mRNA level analysis: Pnlip mRNA levels in the pancreas of the congenic rat $(2.81 \pm 0.21$ for 15 weeks of age and $3.03 \pm 0.43$ for 35 weeks of age) were similar to those of the OLETF rat $(2.98 \pm 0.40$ for 15 weeks of age and $3.13 \pm 0.31$ for 35 weeks of age) but $\sim 3$-fold higher than those of the F344 rat $(0.82 \pm 0.06$ for 15 weeks of age and $0.95 \pm 0.06$ for 35 weeks of age) at both 15 and 35 weeks of age $(\mathrm{p}<0.05)$. The (congenic $\times$ F344) $\mathrm{F}_{1}$ rat $(1.92 \pm 0.20$ for 15 weeks of age and $1.85 \pm 0.19$ for 35 weeks of age) exhibited an intermediate value between the F344 and congenic rats in the pancreatic Pnlip mRNA levels at both 15 and 35 weeks of age and all the differences between the $F_{1}$ and the F344 or congenic were statistically significant $(\mathrm{p}<0.05)$. Further, the normal BN rat showed the similar pancreatic Pnlip mRNA levels to the F344 but their levels were $\sim 3$-fold lower as compared to those of the congenic rat (data not shown). These results indicate that Pnlip possesses an OLETF allele-specific increase of mRNA levels in the pancreas in an additive manner, consistent with the result of phenotype evaluation in the F344, congenic and $F_{1}$ rats. These suggest that the increased mRNA levels of Pnlip may cause the increase of intramuscular fat content in the OLETF rat.

Pnlip polymorphism search: We could not find any polymorphisms between the F344 and OLETF rats in the exon/intron and 3'-flanking regions of Pnlip by the direct 
sequencing. Additionally, no polymorphisms were detected between the F344 and OLETF rats in the Pnlip 5 '-flanking region, except for a $\sim 2.8-\mathrm{kb}$ genomic segment which contains tandem repeats of $\sim 38$-bp core sequence, between the bases 5517 and 2698 upstream from the transcription start site of Pnlip (nucleotide positions 1792587-1795406 of NW_047570). PCR amplification of the $\sim 2.8$-kb genomic segment identified approximately $2.8-\mathrm{kb}$ PCR product in the $\mathrm{F} 344$ rat but about $3.0-\mathrm{kb}$ PCR product in the OLETF and congenic rats. It is likely that the PCR-product length polymorphism is attributable to a variation in the number of repeats of core sequence. Further, the normal BN rat exhibited $\sim 2.8-\mathrm{kb}$ PCR amplification product similar to the F344 rat. These results indicate that the Pnlip OLETF allele is longer in VNTR within the 5'-flanking region than the normal alleles.

A computer scan of a $\sim 2.8 \mathrm{~kb}$ genomic segment (nucleotide positions 1792587-1795406 of NW_047570) containing tandem repeats of core sequence with MOTIF program disclosed dozens of putative binding sites for known transcription factors in the tandem repeat region. Of the notables were binding sites for AP1 and USF1 which are highly expressed and seem to exert a function in the pancreas.

It has been reported that the longer variants of VNTR in the regulatory region of serotonin transporter gene were associated with enhanced mRNA levels of serotonin transporter gene (Lesch et al., 1996). Additionally, the class III longer alleles (140-200 repeats) of VNTR in the regulatory region of Insulin gene (INS) were associated with 2-3 fold higher INS mRNA levels as compared with the class I shorter alleles (23-63 repeats) of which transmission predisposes to type 1 diabetes in human (Vafiadis et al., 1997; Pugliese et al., 1997). The tandem repeat region of $\mathrm{NSS}$ has been reported to also contain binding sites for the transcription factor PURA and to regulate INS transcription (Kennedy et al., 1995). These suggest that the longer OLETF allele in Pnlip VNTR may be associated with the increased Pnlip mRNA levels in the OLETF rat.

Cosegregation of the intramuscular fat content QTL with Pnlip VNTR: We performed genotyping of Pnlip VNTR for the 12 informative recombinants selected from the congenic strain. The genotyping allowed the 12 informative recombinants to be classified into two types, Pnlip VNTR OLETF allele homozygote $(\mathrm{O} / \mathrm{O}, \mathrm{n}=7)$ and Pnlip VNTR heterozygote $(\mathrm{O} / \mathrm{F}, \mathrm{n}=5)$. Allele segregation pattern of the intramuscular fat content QTL in the 12 informative recombinants was completely coincident with that of Pnlip VNTR indicating complete cosegregation of the intramuscular fat content QTL with Pnlip VNTR. This suggests that Pnlip is the QTG for the intramuscular fat content QTL and that the longer allele of Pnlip VNTR may be a causal mutation for phenotype of increase of intramuscular fat content in the OLETF rat. Taken together, the present study might support a hypothesis that the longer allele of Pnlip VNTR causes phenotype of increase of intramuscular fat content through increasing Pnlip mRNA levels in the OLETF rat. There is an evidence for a linkage of marbling trait to genomic region containing PNLIP on bovine chromosome 26 (Takasuga et al., 2007). Based on the present results, it will be important to use PNLIP as candidate gene for marbling trait in bovine association study.

\section{CONCLUSION}

In this study, we suggests that Pnlip encoding pancreatic lipase is the QTG responsible for the intramuscular fat content in the rat. This study will provide an useful information for the understanding of the molecular architecture of marbling that is an economically important trait of beef cattle.

\section{ACKNOWLEDGEMENTS}

This research was supported in part by Grant-in-Aid for Scientific Research on Priority Areas (C) Medical Genome Science from the Ministry of Education, Culture, Sports, Science and Technology of Japan.

\section{REFERENCES}

Boylston, T.D., S.A. Morgan, K.A. Johnson, J.R. Busboom, R.W. Jr Wright and J.J. Reeves, 1995. Lipid content and composition of Wagyu and domestic breeds of beef. J. Agric. Food Chem., 43: 1202-1207.

Busboom, J.R., L.E. Jeremiah, L.L. Gibson, K.A. Johnson, C.T. Gaskins, J.J. Reeves and R.W. Wright, 1993. Effects of biological source on cooking and palatability attributes of beef produced for the Japanese market. Meat Sci., 35: 241-258.

Cameron, P.J., M. Zembayashi, D.K. Lunt, T. Mitsuhashi, M. Mitsumoto, S. Ozawa and S.B. Smith, 1994. Relationship between Japanese beef marbling standard and intramuscular lipid in the $M$. longissimus thoracis of Japanese Black and American Wagyu cattle. Meat Sci., 38: 361-364.

Hill, J.O., J. Hauptman, J.W. Anderson, K. Fujioka and P.M. O'Neil et al., 1999. Orlistat, a lipase inhibitor, for weight maintenance after conventional dieting: A 1-y study. Am. J. Clin. Nutr., 69: 1108-1116. 
Kawano, K., T. Hirashima, S. Mori, Y. Saitoh, M. Kurosumi and T. Natori, 1992. Spontaneous longterm hyperglycemic rat with diabetic complications. Otsuka Long-Evans Tokushima Fatty (OLETF) strain. Diabetes, 41: 1422-1428.

Kennedy, G.C., M.S. German and W.J. Rutter, 1995. The minisatellite in the diabetes susceptibility locus IDDM2 regulates insulin transcription. Nat. Genet., 9: 293-298.

Lesch, K.P., D. Bengel, A. Heils, S.Z. Sabol and B.D. Greenberg et al., 1996. Association of anxietyrelated traits with a polymorphism in the serotonin transporter gene regulatory region. Science, 274: 1527-1531.

Lowe, M.E., 2002. The triglyceride lipases of the pancreas. J. Lipid Res., 43: 2007-2016.

Pugliese, A., M. Zeller, A. Jr Fernandez, L.J. Zalcberg and R.J. Bartlett et al., 1997. The insulin gene is transcribed in the human thymus and transcription levels correlated with allelic variation at the INS VNTR-IDDM2 susceptibility locus for type 1 diabetes. Nat. Genet., 15: 293-297.

Shikamoto, Y. and T. Morita, 1999. Expression of factor X in both the rat brain and cells of the central nervous system. FEBS Lett., 463: 387-389.

Sims, H.F., M.L. Jennens and M.E. Lowe, 1993. The human pancreatic lipase-encoding gene: Structure and conservation of an $A l u$ sequence in the lipase gene family. Gene, 131: 281-285.
Sjostrom, L., A. Rissanen, T. Andersen, M. Boldrin, A. Golay, H.P.F. Koppeschaar and M. Krempf, 1998. Randomised placebo-controlled trial of orlistat for weight loss and prevention of weight regain in obese patients, European Multicentre Orlistat Study Group. Lancet, 352: 167-172.

Takasuga, A., T. Watanabe, Y. Mizoguchi, T. Hirano and N. Ihara et al., 2007. Identification of bovine QTL for growth and carcass traits in Japanese Black cattle by replication and identical-by-descent mapping. Mammalian Genome, 18: 125-136.

Tanomura, H., T. Miyake, Y. Taniguchi, N. Manabe and H. Kose et al., 2002. Detection of a quantitative trait locus for intramuscular fat accumulation using the OLETF rat. J. Vet. Med. Sci., 64: 45-50.

Tanomura, H., Y. Taniguchi, Y. Muramatsu, H. Kose and T. Miyake ., 2003. A congenic strain (F344.OLETF$\mathrm{Imfm}$ ) displays the existence of intramuscular fat accumulation QTL on rat chromosome 1. Exp. Anim., 52: 303-308.

Vafiadis, P., S.T. Bennett, J.A. Todd, J. Nadeau and R. Grabs et al., 1997. Insulin expression in human thymus is modulated by INS VNTR alleles at the IDDM2 locus. Nat. Genet., 15: 289-292.

Wei, S., K. Wei, D.H. Moralejo, T. Yamada, K. Izumi and K. Matsumoto, 1998. An integrated genetic map of the rat with 562 markers from different sources. Mammalian Genome, 9: 1002-1007. 